CTP-TAMU-57/96

hep-th/9611134

\title{
From Topology to Generalised Dimensional Reduction
}

\author{
I.V. Lavrinenko, H. Lü ${ }^{\dagger}$ and C.N. Pope ${ }^{\dagger}$ \\ Center for Theoretical Physics, Texas A\&M University, College Station, Texas 77843
}

\begin{abstract}
$\underline{\text { ABSTRACT }}$
In the usual procedure for toroidal Kaluza-Klein reduction, all the higher-dimensional fields are taken to be independent of the coordinates on the internal space. It has recently been observed that a generalisation of this procedure is possible, which gives rise to lower-dimensional massive supergravities. The generalised reduction involves allowing gauge potentials in the higher dimension to have an additional linear dependence on the toroidal coordinates. In this paper, we show that a much wider class of generalised reductions is possible, in which higher-dimensional potentials have additional terms involving differential forms on the internal manifold whose exterior derivatives yield representatives of certain of its cohomology classes. We consider various examples, including the generalised reduction of M-theory and type II strings on K3, Calabi-Yau and 7-dimensional Joyce manifolds. The resulting massive supergravities support domain-wall solutions that arise by the vertical dimensional reduction of higher-dimensional solitonic $p$-branes and intersecting $p$-branes.
\end{abstract}

\footnotetext{
${ }^{\dagger}$ Research supported in part by DOE Grant DE-FG05-91-ER40633
} 


\section{Introduction}

M-theory or string theory are generally believed to be the fundamental theories that may provide a basis for quantising Einstein's general relativity. One of the reasons for the recent upsurge of interest in the subject has been the discovery of duality relations between string theories that were at one time believed to be independent. One of the ways in which these duality relations can be brought to light is by studying the dimensionally reduced theories that are obtained by compactifying on certain internal spaces. The kind of dimensional reduction that is usually considered is the standard Kaluza-Klein procedure, in which the fields of the higher-dimensional theory are first expanded in terms of complete sets of harmonics on the internal space, followed by a truncation to the massless sector of the resulting lower-dimensional theory. It is crucial that this truncation be consistent, which means that all solutions of the lower-dimensional truncated theory should also be solutions of the higher-dimensional one. Only then will the properties of the lower-dimensional theory reflect themselves in corresponding properties of the higher-dimensional theory. The criterion for consistency is that when one considers the equations of motion for the lowerdimensional massive fields prior to truncation, there should be no source terms constructed purely from the massless fields that are to be retained. This consistency of the truncation is obvious for the case of toroidal compactifications with the standard Kaluza-Klein ansatz, where the higher-dimensional fields are simply taken to be independent of the toroidal coordinates. In more complicated cases such as compactifications on K3 or Calabi-Yau spaces, consistency is not so obvious, since products of the zero-mode harmonics can generate nonzero-mode harmonics. It appears that in fact supersymmetry plays a crucial in establishing the consistency of the truncation in these cases [1, 2].

It was recently observed that the usual Kaluza-Klein ansatz for toroidal compactification is slightly more restrictive than is actually necessary in order to achieve consistency of the truncation. Specifically, it was shown in [3] that in the dimensional reduction of the type IIB theory on a circle, one can allow the Ramond-Ramond axion $\chi$ to have an additional linear dependence on the coordinate $z$ of the circle, $\chi(x, z) \rightarrow m z+\chi(x)$, where $x$ denotes the lower-dimensional coordinates and $m$ is a constant parameter. This does not upset the consistency of the reduction, since $\chi$ appears in the ten-dimensional equations of motion

only through its derivative $d \chi$, and thus there is still no $z$-dependence, even when $m$ is nonzero. The resulting nine-dimensional theory is a massive supergravity, with a cosmological term [3]. In fact, it is T-dual to the theory that one obtains by performing a standard Kaluza-Klein reduction of the massive IIA supergravity [4] on a circle. This generalisation 
of the Kaluza-Klein ansatz for compactification on a circle was subsequently applied to the dimensional reduction of all other $D \leq 11$ maximal supergravities in [5]. (In fact generalised Kaluza-Klein ansätze that give rise to cosmological terms were also discussed in a general group-theoretic framework in [6]. It was also observed in [7, 8], in the context of compactifying the heterotic string to $D=4$, that wrapping the 5 -brane on a 3 -torus to give rise to a membrane in $D=4$ would require some ansatz that went beyond the usual Kaluza-Klein dimensional reduction.)

Consistent generalisations of the Kaluza-Klein ansatz are possible in more complicated compactifications also. In [9], it was shown that the generalised ansatz for the axion $\chi$ discussed above is a special case of the ansatz $A_{n-1}(x, z)=m \omega_{n-1}(z)+$ standard terms, where $A_{n-1}$ is an $(n-1)$-form potential in the higher-dimensional theory, which is then compactified on an $n$-dimensional internal manifold $M_{n}$ with coordinates $z$, whose volume form $\Omega_{n}$ is given locally by $d \omega_{n-1}$. (In the case $M_{1}=S^{1}$, we can represent $\omega_{0}$ locally by $\omega_{0}=z$, giving the globally-defined volume form $\Omega_{1}=d z$.) Again, provided that $A_{n-1}$ appears always through its exterior derivative $d A_{n-1}$ in the higher-dimensional equations of motion, these equations will depend only on the zero-mode harmonics on $M_{n}$ after imposing the generalised ansatz, and thus the truncation will still be consistent.

In all cases, the generalised dimensional reduction procedure gives rise to a massive supergravity with a cosmological term, of the form $-\frac{1}{2} m^{2} e e^{a \phi}$, where $\phi$ is some dilatonic scalar field. Such theories admit no maximally-symmetric Minkowski or anti-de Sitter ground state. Instead, the most symmetrical, and therefore most natural, ground state solution is a domain wall, which is a $(D-2)$-brane in $D$ dimensions. In fact this domain wall solution arises from the vertical dimensional reduction of a standard kind of $p$-brane soliton in the higher dimension. The field strength that supports this higher-dimensional $p$-brane solution has a form that is compatible with the generalised Kaluza-Klein ansatz for its potential. Indeed, it is compatible only with the generalised ansatz, and in fact the original motivation for considering such a generalisation was in order to explain how a 7-brane in the type IIB string could be vertically reduced to a solution of a theory in $D=9$ [3] .

In this paper, we shall develop these ideas further, by showing that one can make use of representatives of some appropriate cohomology classes of the compactifying manifold $M_{n}$, enlarging considerably the number of possibilities for generalised Kaluza-Klein reductions. In section 2, we shall explain the basic idea, and apply it to compactifications of the type IIA string. Then, in section 3, we shall consider applications to the compactification of M-theory, followed by compactifications of the type IIB theory in section 4 . In section 5 , 
we end the paper with a discussion of the supersymmetry of the compactified theories, and duality.

\section{Generalised dimensional reduction of type IIA strings}

We begin our discussion by considering the case of the type IIA string, and its generalised dimensional reduction on certain Ricci-flat compact manifolds $M_{n}$. In particular, we shall consider its compactification on $T^{4}, \mathrm{~K} 3$ and six-dimensional Calabi-Yau spaces. The lowenergy limit of the type IIA string is type IIA supergravity, whose bosonic sector contains the metric and dilaton, together with a rank 4, a rank 3 and a rank 2 field strength. We shall denote these by $g_{M N}, \phi_{1}, F_{4}, F_{3}^{(1)}$ and $\mathcal{F}_{2}^{(1)}$ respectively. This notation is derived from the fact that type IIA supergravity itself can be obtained by dimensional reduction from $D=11$ supergravity. Using the notation introduced in [10], the toroidal reduction from

$D=11$ to $D$ dimensions gives field strengths $F_{4}, F_{3}^{(i)}, F_{2}^{(i j)}, F_{1}^{(i j k)}$ from the 4 -form $F_{4}$ in $D=11$, and field strengths $\mathcal{F}_{2}^{(i)}$ and $\mathcal{F}_{1}^{(i j)}$ from the $D=11$ metric. In addition, there will be dilatonic scalars $\phi_{i}$. The index $i$ runs over the $11-D$ toroidally-compactified coordinates $z_{i}$.

Using the above notation, the bosonic sector of type IIA supergravity becomes

$$
\begin{aligned}
\hat{e}^{-1} \hat{\mathcal{L}}= & \hat{R}-\frac{1}{2}\left(\partial \phi_{1}\right)^{2}-\frac{1}{48} e^{-\frac{1}{2} \phi_{1}} \hat{F}_{4}^{2}-\frac{1}{12} e^{\phi_{1}}\left(\hat{F}_{3}^{(1)}\right)^{2}-\frac{1}{4} e^{-\frac{3}{2} \phi_{1}}\left(\hat{\mathcal{F}}^{(1)}\right)^{2} \\
& +\frac{1}{2} \hat{e}^{-1} d \hat{A}_{3} \wedge d \hat{A}_{3} \wedge \hat{A}_{2}^{(1)}
\end{aligned}
$$

where the final term is presented as a 10-form. The field strengths are given by

$$
\hat{F}_{4}=d \hat{A}_{3}+\hat{A}_{2}^{(1)} \wedge d \hat{\mathcal{A}}_{1}^{(1)}, \quad \hat{F}_{3}^{(1)}=d \hat{A}_{2}^{(1)}, \quad \hat{\mathcal{F}}_{2}^{(1)}=d \hat{\mathcal{A}}_{1}^{(1)}
$$

\section{$2.1 \quad T^{4}$ and $\mathrm{K} 3$ compactifications}

The standard $T^{4}$ compactification, using the usual Kaluza-Klein ansatz, gives rise to maximal $N=4$ supergravity in $D=6$. Various generalisations of this reduction are possible, in which a higher-dimensional gauge potential acquires an additional linear dependence on one or more of the coordinates of the 4-torus. These generalised reductions give rise to massive supergravities in $D=6$, with cosmological terms. One example that has recently been discussed involves taking the ansatz for the 3 -form potential in $D=10$ to have the generalised form

$$
A_{3}^{(1)}(x, z)=m \omega_{3}+A_{3}^{(1)}(x)+\cdots,
$$


where $d \omega_{3}=\Omega_{4}=d z_{2} \wedge d z_{3} \wedge d z_{4} \wedge d z_{5}$ is the volume form on the 4-torus [9]. One may choose to write $\omega_{3}$ locally as, for example, $\omega_{3}=z_{2} d z_{3} \wedge d z_{4} \wedge d z_{5}$. All the other ten-dimensional fields are reduced using the standard Kaluza-Klein ansatz, in which there is no dependence on any of the $z_{i}$ coordinates. This generalised dimensional reduction gives rise to a massive maximal supergravity in $D=6$ with a cosmological term. Its natural ground state is a domain wall solution, which is the vertical dimensional reduction of the solitonic 4-brane in $D=10$. Note that this massive supergravity theory does not have any ground state that admits the full set of 32 Killing spinors that arise in the Minkowski ground state of massless $N=4$ supergravity in $D=6$. (Nor does it admit an anti-de Sitter ground state, since the cosmological term has a dilaton coupling.) The domain wall ground state admits 16 Killing spinors. Even though there is no maximally-symmetric ground state in the massive theory, we shall nevertheless adopt the standard practice of describing this solution as preserving one half of the global supersymmetry.

The generalised reduction discussed above uses the cohomology class $H^{4}\left(M_{4}, \mathbb{R}\right)$, representing the fact that the volume form $\Omega_{4}$ has a non-zero integral over the entire 4-manifold. In this section, we consider another type of ansatz for the generalised reduction, which makes use of the cohomology class $H^{2}\left(M_{4}, \mathbb{R}\right)$. We shall apply this idea in two examples, beginning with the 4-torus, and then K3. The generalised ansatz will now be made for the 1-form potential rather than the 3-form. In the case of the torus, there are six independent 2 -forms $d z_{i} \wedge d z_{j}$ in $H^{2}\left(T^{4}, \mathbb{R}\right)$, where $2 \leq i<j \leq 5$, allowing a six-parameter family of generalised ansätze for the 1-form potential. We shall consider the following two-parameter example,

$$
\mathcal{A}_{1}^{(1)}(x, z)=m_{1} z_{2} d z_{3}+m_{2} z_{4} d z_{5}+\mathcal{A}_{1}^{(1)}(x)+\mathcal{A}_{0}^{(1 j)} d z_{j}
$$

where the summation in the final term is over $2 \leq j \leq 5$. All the other ten-dimensional fields are reduced using the usual $z$-independent ansätze,

$$
\begin{aligned}
A_{3}(x, z) & =A_{3}(x)+A_{2}^{(i)}(x) \wedge d z_{i}+\frac{1}{2} A_{1}^{(i j)} \wedge d z_{i} \wedge d z_{j}+\frac{1}{6} A_{0}^{(i j k)}(x) d z_{i} \wedge d z_{j} \wedge d z_{k} \\
A_{2}^{(1)}(x, z) & =A_{2}^{(1)}(x)+A_{1}^{(1 j)}(x) \wedge d z_{j}+\frac{1}{2} A_{0}^{(1 j k)}(x) d z_{j} \wedge d z_{k}
\end{aligned}
$$

where the indices run over the range 2 to 5 . The metric ansatz is

$$
\begin{aligned}
d s_{10}^{2}= & e^{2 \alpha_{2} \phi_{2}+\cdots 2 \alpha_{5} \phi_{5}} d s_{6}^{2}+e^{2 \alpha_{3} \phi_{3}+\cdots 2 \alpha_{5} \phi_{5}-14 \alpha_{2} \phi_{2}} h_{5}^{2}, \\
& +e^{2 \alpha_{4} \phi_{4}+2 \alpha_{5} \phi_{5}-12 \alpha_{3} \phi_{3}} h_{4}^{2}+e^{2 \alpha_{5} \phi_{5}-10 \alpha_{4} \phi_{4}} h_{3}^{2}+e^{-8 \alpha_{5} \phi_{5}} h_{2}^{2},
\end{aligned}
$$

\footnotetext{
${ }^{1}$ Since the 4-torus also has non-vanishing $H^{3}$ cohomology, we could also consider generalising the ansatz for the 2 -form potential in $D=10$. We shall not pursue this further, since it does not extend to the K3 compactification.
} 
where $\left(\alpha_{i}\right)^{-2}=2(10-i)(9-i)$ and the scalars $\phi_{i}$ and the metric $d s_{6}^{2}$ are functions only of the $x$ coordinates. The 1 -forms $h_{i}$ are given by

$$
h_{i}=d z_{i}+\mathcal{A}_{1}^{(i)}(x)+\mathcal{A}_{0}^{(i j)}(x) d z_{j}
$$

where the summation is over $i<j \leq 5$, since $\mathcal{A}_{0}^{(i j)}$ is defined only for $i<j$. This can easily be inverted to express $d z_{i}$ in terms of $h_{i}$ [10]:

$$
d z_{i}=\gamma_{i j}\left(h_{j}-\mathcal{A}_{1}^{(j)}\right)
$$

where the summation is over $i \leq j \leq 5$, and

$$
\gamma_{i j}=\left[\left(1+\mathcal{A}_{0}\right)^{-1}\right]^{i j}=\delta^{i j}-\mathcal{A}_{0}^{(i j)}+\mathcal{A}_{0}^{(i k)} \mathcal{A}_{0}^{(k j)}+\cdots
$$

Substituting all the above ansätze into the Lagrangian (2.1), we obtain a six-dimensional Lagrangian that describes a consistent truncation of type IIA supergravity, since it is independent of the $z$ coordinates. The full expression for the six-dimensional Lagrangian is quite complicated, and we shall not present it explicitly here. Instead, we shall concentrate on the essential features, in particular describing the spectrum of massive and massless fields. When the substitution of the ansätze is performed, one first obtains an expression in $D=6$ with a kinetic term for each of the field strengths formed from the six-dimensional potentials. However, these field strengths will in general have Chern-Simons modifications. To see how these arise, it is simplest to express the ten-dimensional field strengths in terms of a tangent-space basis, since then the expressions for their kinetic terms appearing in (2.1) can immediately be read off. Thus for example, it follows from (2.6) that the 3 -form field strength in $D=10$ becomes

$$
F_{3}^{(1)}(x, z)=d A_{2}^{(1)}(x)+d A_{1}^{(1 j)}(x) \wedge d z_{j}+\frac{1}{2} d A_{0}^{(1 j k)} d z_{j} \wedge d z_{k},
$$

which can then be expressed in the tangent-space basis by replacing $d z_{i}$ by $h_{i}$, using (2.9). For the purposes of determining the spectrum of massive and massless fields in $D=6$, it suffices to look only at the terms up to linear order in fields, since these will govern the form of the kinetic terms. Thus we find that all of the field strengths in $D=6$ are given by $F=d A+2$ 'nd order terms, with the following exceptions:

$$
\begin{array}{lcc}
F_{2}^{(23)} \sim d A_{1}^{(23)}+m_{1} A_{2}^{(1)}, & F_{2}^{(45)} \sim d A_{1}^{(45)}+m_{2} A_{2}^{(1)}, \\
F_{1}^{(234)} \sim d A_{0}^{(234)}+m_{1} A_{1}^{(14)}, & F_{1}^{(235)} \sim d A_{0}^{(235)}+m_{1} A_{1}^{(15)}, \\
F_{1}^{(245)} \sim d A_{0}^{(245)}+m_{2} A_{1}^{(12)}, & F_{1}^{(345)} \sim d A_{0}^{(345)}+m_{2} A_{1}^{(13)}, \\
\mathcal{F}_{1}^{(12)} \sim d \mathcal{A}_{0}^{(12)}+m_{1} \mathcal{A}_{1}^{(3)}, & \mathcal{F}_{1}^{(13)} \sim d \mathcal{A}_{0}^{(13)}-m_{1} \mathcal{A}_{1}^{(2)}, \\
\mathcal{F}_{1}^{(14)} \sim d \mathcal{A}_{0}^{(14)}+m_{2} \mathcal{A}_{1}^{(5)}, & \mathcal{F}_{1}^{(15)} \sim d \mathcal{A}_{0}^{(15)}+m_{2} \mathcal{A}_{1}^{(4)} .
\end{array}
$$


Here, the $\sim$ symbol indicates that we have omitted the terms of 2'nd order and above.

We can see from (2.12) that, at least to the leading order, the potentials appearing through their exterior derivatives on the right-hand sides can be absorbed by making gauge transformations of the associated undifferentiated potentials. After doing this, the previous kinetic terms for the fields that are absorbed will become mass terms for the potentials that absorb them. In other words, the former fields are eaten by the latter, in order for them to become massive. Consistency of the theory requires that the fields that are eaten should then disappear everywhere from the Lagrangian. To clarify this phenomenon, and to show that it works to all orders in the various fields, we may exhibit explicitly the relevant local symmetries of the Lagrangian in $D=6$ that can be used to eliminate the fields. These symmetries originate from gauge symmetries of the ten-dimensional theory. After the generalised dimensional reduction, they become Stückelburg symmetries in $D=6$, under which the fields that are eaten undergo pure non-derivative shift symmetries. The symmetries can therefore be used to set these fields to zero.

The relevant local symmetries in $D=10$ are included among the gauge transformations of the potentials $\hat{A}_{3}, \hat{A}_{2}^{(1)}$ and $\hat{\mathcal{A}}_{1}^{(1)}$, and certain general coordinate transformations of the compactified coordinates:

$$
\begin{aligned}
\hat{A}_{3} & \rightarrow \hat{A}_{3}^{\prime}=\hat{A}_{3}+d \hat{\Lambda}_{2}-\hat{\Lambda}_{1}^{(1)} \wedge d \hat{\mathcal{A}}_{1}^{(1)}, \\
\hat{A}_{2}^{(1)} & \rightarrow \hat{A}_{2}^{(1)}=\hat{A}_{2}^{(1)}+d \hat{\Lambda}_{1}^{(1)} \\
\hat{\mathcal{A}}_{1}^{(1)} & \rightarrow \hat{\mathcal{A}}_{1}^{(1)^{\prime}}=\hat{\mathcal{A}}_{1}^{(1)}+d \hat{\Lambda}_{0}^{(1)}, \\
z_{i} & \rightarrow z_{i}^{\prime}=z_{i}+\xi_{i}(x) .
\end{aligned}
$$

The gauge parameters are dimensionally reduced in the same way as the gauge potentials, namely $\hat{\Lambda}_{1}^{(1)}=\Lambda_{1}^{(1)}(x)+\Lambda_{0}^{(1 j)}(x) d z_{j}$, etc.

Consider first the lower-dimensional gauge parameters $\Lambda_{1}^{(1)}(x)$. These generate the transformations

$$
\begin{array}{ll}
A_{1}^{(23)^{\prime}}=A_{1}^{(23)}-m_{1} \Lambda_{1}^{(1)}, & A_{1}^{(45)^{\prime}}=A_{1}^{(45)}-m_{2} \Lambda_{1}^{(1)}, \\
A_{3}^{\prime}=A_{3}-\Lambda_{1}^{(1)} \wedge d \mathcal{A}_{1}^{(1)}, & A_{2}^{(1)^{\prime}}=A_{2}^{(1)}+d \Lambda_{1}^{(1)}, \\
A_{2}^{(i)^{\prime}}=A_{2}^{(i)}-\Lambda_{1}^{(1)} \wedge d \mathcal{A}_{0}^{(1 i)}, & i=2, \ldots, 5,
\end{array}
$$

where we have displayed only those fields on which the transformation acts non-trivially. We see that the ten-dimensional gauge symmetry associated with the gauge parameter $\Lambda_{1}^{(1)}$ becomes a Stückelburg symmetry for the potentials $A_{1}^{(23)}$ and $A_{1}^{(45)}$. Thus we can choose 
the parameter $\Lambda_{1}^{(1)}$ appropriately so as to set either $A_{1}^{(23)}$ or $A_{1}^{(45)}$, but not both, to zero. Either way, it has the consequence that the potential $A_{2}^{(1)}$ becomes massive.

Analogously, the low-dimensional gauge parameters $\Lambda_{0}^{(1 i)}(x)$, for $i=2, \ldots, 5$, also generate Stückelburg symmetries:

$$
\begin{aligned}
& A_{0}^{(i 23)^{\prime}}=A_{0}^{(i 23)}-m_{1} \Lambda_{0}^{(1 i)}, \quad i=4,5, \quad A_{0}^{(i 45)^{\prime}}=A_{0}^{(i 45)}-m_{2} \Lambda_{0}^{(1 i)}, \quad i=2,3, \\
& A_{1}^{(i j)^{\prime}}=A_{1}^{(i j)}+\Lambda_{0}^{(1 i)} d \mathcal{A}_{0}^{(1 j)}-\Lambda_{0}^{(1 j)} d \mathcal{A}_{0}^{(1 i)}, \quad i, j=2, \ldots, 5 \\
& A_{2}^{(i)}=A_{2}^{(i)}-\Lambda_{0}^{(1 i)} d \mathcal{A}_{1}^{(1)}, \quad i=2, \ldots, 5
\end{aligned}
$$

Thus we can set the potentials $A_{0}^{(234)}, A_{0}^{(235)}, A_{0}^{(245)}$ and $A_{0}^{(345)}$ to zero and correspondingly the fields $A_{1}^{(1 i)}$ become massive, with masses $m_{1}$ for $i=4,5$ and $m_{2}$ for $i=2,3$.

The Stückelburg symmetry that can be used to eliminate the potentials $\mathcal{A}_{0}^{1 i}$ comes from the general coordinate transformations $z_{i}^{\prime}=z_{i}+\xi_{i}(x)$ in $D=10$. Since these leave the left-hand sides of the ten-dimensional expansions (2.5), (2.6) and (2.8) invariant, we can read off their action on the six-dimensional fields:

$$
\begin{aligned}
& \mathcal{A}_{0}^{(12)}=\mathcal{A}_{0}^{(12)}+m_{1} \xi_{3}, \quad \mathcal{A}_{0}^{(13)^{\prime}}=\mathcal{A}_{0}^{(13)}-m_{1} \xi_{2} \\
& \mathcal{A}_{0}^{(14)^{\prime}}=\mathcal{A}_{0}^{(12)}+m_{2} \xi_{5}, \quad \mathcal{A}_{0}^{(15)^{\prime}}=\mathcal{A}_{0}^{(15)}-m_{1} \xi_{4} \\
& A_{1}^{(i j)^{\prime}}=A_{1}^{(i j)}-A_{0}^{(i j k)} d \xi_{k}, \quad \mathcal{A}_{1}^{(1)^{\prime}}=\mathcal{A}_{1}^{(1)}-\mathcal{A}_{0}^{(1 i)} d \xi_{i}-m_{1} \xi_{3} d \xi_{2}-m_{2} \xi_{5} d \xi_{4} \\
& \mathcal{A}_{1}^{(i)^{\prime}}=\mathcal{A}_{1}^{(i)}-d \xi_{i}-\mathcal{A}_{0}^{(i j)} d \xi_{j}, \quad i=2, \ldots, 5, \quad i \leq j \leq 5, \\
& A_{2}^{(1)^{\prime}}=A_{2}^{(1)}-A_{1}^{(1 i)} \wedge d \xi_{i}+\frac{1}{2} A_{0}^{(1 i j)} d \xi_{i} \wedge d \xi_{j}, \\
& A_{2}^{(i)^{\prime}}=A_{2}^{(i)}+A_{1}^{(i j)} \wedge d \xi_{i}+\frac{1}{2} A_{0}^{(i j k)} d \xi_{j} \wedge d \xi_{k}, \quad i=2, \ldots, 5 \\
& A_{3}^{\prime}=A_{3}-A_{2}^{(i)} \wedge d \xi_{i}+\frac{1}{2} A_{1}^{(i j)} \wedge d \xi_{i} \wedge d \xi_{j}-\frac{1}{6} A_{0}^{(i j k)} d \xi_{i} \wedge d \xi_{j} \wedge d \xi_{k} .
\end{aligned}
$$

In summary, we have seen that the generalised Kaluza-Klein ansatz (2.5) gives masses to the fields $A_{2}^{(1)}, A_{1}^{(1 i)}$ and $\mathcal{A}_{1}^{(i)}$. The fields that are eaten in each case can be seen by inspection of (2.12). In addition, we can see from the expansion of (2.5) up to linear order that the four axions $\mathcal{A}_{0}^{(24)}, \mathcal{A}_{0}^{(25)}, \mathcal{A}_{0}^{(34)}$ and $\mathcal{A}_{0}^{(35)}$ acquire masses $m_{1}$. Since these are scalars, there is no associated Stückelburg symmetry. There are two cosmological terms in $D=6$, taking the form

$$
\mathcal{L}_{\text {cosmo }}=-\frac{1}{2} m_{1}^{2} e e^{\vec{b}_{123} \cdot \vec{\phi}}-\frac{1}{2} m_{2}^{2} e e^{\vec{b}_{145} \cdot \vec{\phi}}
$$

where the dilaton vectors $\vec{b}_{123}$ and $\vec{b}_{145}$ are defined in [5].

As we have discussed previously, this massive theory in $D=6$ will admit domain wall solutions. The metric takes the form

$$
d s_{6}^{2}=\left(H_{1} H_{2}\right)^{\frac{1}{4}} d x^{\mu} d x^{\nu} \eta_{\mu \nu}+\left(H_{1} H_{2}\right)^{\frac{5}{4}} d y^{2},
$$


where $H_{1}=1+m_{1}|y|$ and $H_{2}=1+m_{2}|y|$. There are two dilatonic scalars, $\varphi_{1}=\vec{b}_{123} \cdot \vec{\phi}$ and $\varphi_{2}=\vec{b}_{145} \cdot \vec{\phi}$, given by

$$
e^{-\varphi_{1}}=H_{1}^{\frac{13}{4}} H_{2}^{\frac{5}{4}}, \quad e^{-\varphi_{2}}=H_{2}^{\frac{13}{4}} H_{1}^{\frac{5}{4}}
$$

The other orthogonal components of $\vec{\phi}$ are zero. This solution can be oxidised back to $D=10$, where the ten-dimensional metric becomes

$$
\begin{aligned}
d s_{10}^{2}= & \left(H_{1} H_{2}\right)^{-\frac{1}{8}} d x^{\mu} d x^{\nu} \eta_{\mu \nu}+\left(H_{1} H_{2}\right)^{\frac{7}{8}} d y^{2} \\
& +H_{1}^{\frac{7}{8}} H_{2}^{-\frac{1}{8}}\left(d z_{2}^{2}+d z_{3}^{2}\right)+H_{1}^{-\frac{1}{8}} H_{2}^{\frac{7}{8}}\left(d z_{4}^{2}+d z_{5}^{2}\right)
\end{aligned}
$$

with the dilaton $\phi_{1}$ and the 2 -form field strength $\hat{F}_{2}^{(1)}$ given by

$$
e^{\phi_{1}}=\left(H_{1} H_{2}\right)^{3 / 4}, \quad \hat{F}_{2}^{(1)}=m_{1} d z_{2} \wedge d z_{3}+m_{2} d z_{4} \wedge d z_{5}
$$

The metric 2.21 describes two intersecting 6-branes in $D=10$. To see this, we note that if we set $m_{1}=0$, so that the harmonic function $H_{1}$ equals one, the metric describes 6 -branes with world volume coordinates $\left(x^{\mu}, z_{2}, z_{3}\right)$ and transverse space $\left(y, z_{4}, z_{5}\right)$. Since the 6-brane solution is independent of the transverse coordinates $\left(z_{4}, z_{5}\right)$, it describes a plane of 6-branes whose charges are uniformly distributed over $\left(z_{4}, z_{5}\right)$. On the other hand, if we instead set $m_{2}=0$, and hence $H_{2}=1$, the solution describes a plane of 6 -branes distributed over $\left(z_{2}, z_{3}\right)$, with world volume coordinates $\left(x^{\mu}, z_{4}, z_{5}\right)$. If we now consider the general case where $m_{1}$ and $m_{2}$ take generic non-vanishing values, we see that the solution (2.21) interpolates between these two configurations, and can be interpreted as describing the intersection of the two six-branes. Many other examples of intersecting branes were discussed in [11-16].

So far we have discussed two examples of generalised compactification of the type IIA theory on a 4-torus. The first one involves a generalised ansatz for the 3 -form potential, given by (2.4), and the second involves a generalised ansatz for the 1-form potential, given in (2.5). In fact we can make both the generalised ansätze simultaneously without spoiling the consistency of the Kaluza-Klein reduction, since the 3 -form potential $\hat{A}_{3}$ and the 1 form potential $\hat{\mathcal{A}}_{1}^{(1)}$ simultaneously appear in the Lagrangian only through their exterior derivatives. Furthermore, the ansatz for the 1-form potential given in (2.5) is just an example for the generalised reduction. The most general ansatz takes the form $\hat{\mathcal{A}}_{1}^{(1)}=$ $m_{i j} z_{i} d z_{j}+\cdots$

One reason that we have chosen the specific example of the ansatz (2.5) is that the associated reduced theory in $D=6$ admits a domain-wall solution, given by (2.19); not 
all the generalised reductions of the type IIA theory admit $p$-brane solutions. Another reason is that the above example, which is a generalised Kaluza-Klein compactification on a 4-torus, can easily be extended to a compactification on the K3 manifold. To see this we note that in the metric describing two intersecting 6-branes (2.21), the 4-dimensional compactified space $\left(z_{2}, z_{3}, z_{4}, z_{5}\right)$ becomes isotropic when $m_{1}=m_{2}$, and the 2-form field strength given in (2.22) becomes self-dual with respect to the four compactified dimensions. In fact, modulo relabellings of the internal coordinates $z_{i}$, the configuration $(2.22)$ for $\hat{F}_{2}^{(1)}$, with two terms spanning the internal 4-dimensional space, is the unique possibility that can give the isotropic form for the internal metric. Thus we will still have a solution if we replace the compactifying 4-torus with metric $d z_{i} d z_{i}$ by any compact 4-manifold with Ricci-flat metric $d s_{4}^{2}$ which admits a covariantly constant self-dual 2-form $J$, giving

$$
\begin{aligned}
d s_{10}^{2} & =H^{-\frac{1}{4}} d x^{\mu} d x^{\nu} \eta_{\mu \nu}+H^{\frac{7}{4}} d y^{2}+H^{\frac{3}{4}} d s_{4}^{2}, \\
e^{\phi_{1}} & =H^{\frac{3}{4}}, \quad \hat{F}_{2}^{(1)}=m J
\end{aligned}
$$

where $H=1+m|y|$. In particular, we may take the compactifying space to be K3. Note that when $H_{1}=H_{2}=H$, the six-dimensional domain-wall solution (2.19) is of the form 17

$$
d s_{6}^{2}=H^{\frac{4}{\Delta(D-2)}} d x_{\mu} d x_{\nu} \eta_{\mu \nu}+H^{\frac{4(D-1)}{\Delta(D-2)}} d y^{2}
$$

with $D=6$ and $\Delta=2$. In general, the metrics (2.24) are domain-wall solutions for the single-scalar Lagrangian

$$
e^{-1} \mathcal{L}=R-\frac{1}{2}(\partial \phi)^{2}-\frac{1}{2} m^{2} e^{a \phi},
$$

with $a$ parameterised by $a^{2}=\Delta+2(D-1) /(D-2)$ [17]. This Lagrangian can be obtained as a consistent truncation of a Lagrangian involving multiple scalars and cosmological terms, in a manner described in [10]. Domain-wall solutions in 4-dimensional supergravities were studied earlier in [18, 19, 20]. Type II domain walls in $D=10$ were related to D8-branes in [21]. Domain-wall structures in generic dimensions and their dimensional reduction were studied in 22].

The above dimensional reduction of the ten-dimensional solution on K3 may be implemented also at the level of the supergravity theory itself. This generalised reduction procedure exploits the cohomology class $H^{2}\left(M_{4}, \mathbb{R}\right)$ of the compactifying 4-manifold $M_{4}$. Whereas for $T^{4}$ it has dimension 6, corresponding to three self-dual and three anti-self-dual harmonic 2-forms, for $\mathrm{K} 3$ it has dimension 22 . These comprise 3 self-dual covariantlyconstant 2 -forms $\Omega_{2+}^{(i)}$, one of which is the Kähler form, and 19 anti-self-dual harmonic 
2-forms $\Omega_{2-}^{(\alpha)}$, which are not covariantly constant. For simplicity, we shall consider the case where we choose just one of the three covariantly-constant self-dual 2-forms, say $\Omega_{2+}^{(3)}$, for the generalised reduction. Locally, we may write $\Omega_{2+}^{(3)}=d w$, and the ansätze for the ten-dimensional potentials are

$$
\begin{aligned}
\hat{\mathcal{A}}_{1}^{(1)}(x, z) & =m \omega+\mathcal{A}^{(1)}(x), \\
\hat{A}_{3}(x, z) & =A_{3}(x)+\sum_{i=1}^{3} A_{1}^{(i)}(x) \wedge \Omega_{2+}^{(i)}+\sum_{\alpha=1}^{19} A_{1}^{(\alpha)}(x) \wedge \Omega_{2-}^{(\alpha)}, \\
\hat{A}_{2}^{(1)}(x, z) & =A_{2}^{(1)}(x)+\sum_{i=1}^{3} A_{0}^{(1 i)}(x) \Omega_{2+}^{(i)}+\sum_{\alpha=1}^{19} A_{0}^{(1 \alpha)}(x) \Omega_{2-}^{(\alpha)} .
\end{aligned}
$$

The ansatz for the metric will be the standard one, with the 58 parameters for Ricci-flat metrics on K3 becoming $x$-dependent six-dimensional scalar fields. Note that there are no vector potentials coming from the metric, since the first Betti number of K3 is zero.

The spectrum of massive and massless fields can be determined in the same manner as we did previously for the toroidal compactification. Substituting the ansätze (2.26) into (2.3), we see from an examination of the terms linear in fields that $A_{2}^{(1)}$ becomes massive, by eating the field $A_{1}^{(i)}$ with $i=3$, and also the axion $A_{0}^{(1 i)}$ with $i=3$ becomes massive. (The last result follows because $\Omega_{2-}^{(\alpha)} \wedge \Omega_{2+}^{(i)}=0$ and $\Omega_{2+}^{(i)} \wedge \Omega_{2+}^{(j)}=2 \Omega_{4} \delta^{i j}$.) All the other six-dimensional fields will be massless. There is also a cosmological term coming from the kinetic term for the ten-dimensional 2-form field strength. The theory in $D=6$ has $N=1$ supersymmetry.

Note that in the K3 compactification that we have been discussing, we chose one of the covariantly-constant harmonic 2 -forms for the generalised reduction procedure. In principle, we could instead choose one of the remaining 19 anti-self-dual harmonic 2-forms, which will not be covariantly constant. This will also give rise to a massive supergravity in 6 dimensions. However, it was evident in our previous discussion of the reduction of the intersecting 5-brane solution in $D=10$ that the covariant-constancy of the harmonic 2-form played an essential role in the solution. Thus a generalised reduction using an harmonic form that is not covariantly constant will give rise to a massive supergravity that does not admit domain-wall solutions of the kind we are considering in this paper. Thus here, and in the generalised reductions in subsequent sections, we shall concentrate on those associated with covariantly-constant harmonic forms. 


\section{$2.2 \quad T^{6}$ and Calabi-Yau compactifications}

The 6-torus has non-vanishing cohomology $H^{p}\left(T^{6}, \mathbb{R}\right)$ for all $0 \leq p \leq 6$. On the other hand, Calabi-Yau manifolds of real dimension 6 have non-vanishing cohomology for $p=0,2,3,4,6$. Thus in either case we may consider generalised reductions based on the cohomology classes $H^{2}\left(M_{6}, \mathbb{R}\right), H^{3}\left(M_{6}, \mathbb{R}\right)$ or $H^{4}\left(M_{6}, \mathbb{R}\right)$. The procedure for implementing the generalised dimensional reduction is similar to the one we described in section (2.1), with an additional term of the form $m \omega$ added to the relevant ten-dimensional potential, where $d \omega$ represents the non-trivial 2'nd, 3'rd or 4'th cohomology. The spectrum of massive fields in the dimensionally-reduced theory is again governed by the structure of the $m$-dependent bilinear terms in the four-dimensional Lagrangian. For example, if we make a generalised ansatz for $\hat{\mathcal{A}}_{1}^{(1)}$, we find that the field $A_{2}^{(1)}$ becomes massive, as do certain axionic fields. The resulting theory is a massive supergravity in $D=4$, with $N=8$ supersymmetry in the case of a toroidal compactification, and $N=2$ in the Calabi-Yau case. Note that if instead the ansatz for $\hat{A}_{3}$ or $\hat{A}_{2}^{(1)}$ is generalised, there will also be bilinear terms in the $D=4$ Lagrangian coming from $d \hat{A}_{3} \wedge d \hat{A}_{3} \wedge \hat{A}_{2}^{(1)}$ in $D=10$. These are associated with topological mass terms.

Having obtained the massive supergravities in $D=4$ via various generalised dimensional procedures, it is interesting to study their vacuum solutions, namely the domain walls. Note that unlike the cases in $D=6$ discussed in the previous subsection, in order to have a domain wall solution in $D=4$ it is not actually essential to make a generalised compactification. This is because a 4 -form field strength in $D=4$ is dual to a cosmological term, and hence can be used to construct an electric domain wall, which is nothing but the vertical dimensional reduction of the membrane in $D=10$. The compactifying space can be any Ricci-flat 6-manifold. However, if we do instead consider the generalised ansatz for the field strength, the membrane in $D=10$ will not survive the compactification since the solution is incompatible with the generalised ansatz, and the domain wall solutions in $D=4$, which are solitonic, arise as solutions from the cosmological terms. These domain-wall solutions in $D=4$ massive supergravities have their origins as intersecting solitonic $p$-branes in $D=10$, as we explained in the examples of $T^{4}$ or K3 compactifications in section 2.1. We shall first discuss the $T^{6}$ compactification, and then show that it can easily be extended to a Calabi-Yau compactification. We start with a generalised dimensional reduction where the 1-form field potential acquires an extra term, corresponding to $\hat{F}_{2}^{(1)}=\Omega_{2}+\cdots$, with

$$
\Omega_{2}=m_{1} d z_{2} \wedge d z_{3}+m_{2} d z_{4} \wedge d z_{5}+m_{3} d z_{6} \wedge d z_{7}
$$


In $D=10$, there exists a solution of three intersecting 6 -branes, with the 2 -form field strength given by (2.27). The metric and the dilaton $\phi_{1}$ of the solution are given by

$$
\begin{aligned}
d s_{10}^{2}= & \left(H_{1} H_{2} H_{3}\right)^{-\frac{1}{8}} d x^{\mu} d x^{\nu} \eta_{\mu \nu}+\left(H_{1} H_{2} H_{3}\right)^{\frac{7}{8}} d y^{2}+H_{1}^{\frac{7}{8}}\left(H_{2} H_{3}\right)^{-\frac{1}{8}}\left(d z_{2}^{2}+d z_{3}^{2}\right) \\
& +H_{2}^{\frac{7}{8}}\left(H_{1} H_{3}\right)^{-\frac{1}{8}}\left(d z_{4}^{2}+d z_{5}^{2}\right)+H_{3}^{\frac{7}{8}}\left(H_{1} H_{2}\right)^{-\frac{1}{8}}\left(d z_{6}^{2}+d z_{7}^{2}\right) \\
e^{\phi_{1}}= & \left(H_{1} H_{2} H_{3}\right)^{\frac{3}{4}}
\end{aligned}
$$

where $H_{\alpha}=1+m_{\alpha}|y|$. This solution can be compactified on the 6 -torus, parameterised by $z^{i}$ for $2 \leq i \leq 7$, giving rise to a domain-wall solution in $D=4$ :

$$
\begin{aligned}
d s_{4}^{2} & =\left(H_{1} H_{2} H_{3}\right)^{\frac{1}{2}}+\left(H_{1} H_{2} H_{3}\right)^{\frac{3}{2}} d y^{2}, \\
e^{-\frac{1}{2} \varphi_{\alpha}} & =H_{\alpha}\left(H_{1} H_{2} H_{3}\right)^{\frac{3}{4}},
\end{aligned}
$$

where $\varphi_{\alpha}=\vec{c}_{a} \cdot \vec{\phi}$, with $\vec{c}_{1}=\vec{b}_{123}, \vec{c}_{2}=\vec{b}_{145}$ and $\vec{c}_{3}=\vec{b}_{167}$. The domain wall (2.29) arises as a solution of the 4-dimensional Lagrangian

$$
e^{-1} \mathcal{L}=R-\frac{1}{2}(\partial \vec{\phi})^{2}-\frac{1}{2} \sum_{\alpha=1}^{3} m_{\alpha}^{2} e^{\vec{c}_{\alpha} \cdot \vec{\phi}}
$$

which can be obtained from the generalised dimensional reduction of the theory.

In order to extend the above discussion to the generalised Calabi-Yau compactification, we can consider the special case where the parameters $m_{\alpha}$ are all equal. In this case, in the solution of the three intersecting 6 -branes in $D=10$, the 2 -form field strength is taken to be proportional to the Kähler form $J$ and the metric becomes

$$
d s_{10}^{2}=H^{-\frac{3}{8}} d x^{\mu} d x^{\nu} \eta_{\mu \nu}+H^{\frac{21}{8}} d y^{2}+H^{\frac{5}{8}} d s_{6}^{2},
$$

where $d s_{6}^{2}$ is the metric of the Calabi-Yau manifold. As in the compactification on the 4-torus that we discussed previously, the form of the three-term expression (2.27) for the 2-form field strength is uniquely singled out, modulo coordinate relabellings, by the requirement that it should give rise to an isotropic form for the six-dimensional internal metric when the charges are set equal. Thus compactifying this solution on the Calabi-Yau manifold gives rise to a single-scalar domain-wall solution in $D=4$ with $\Delta=4 / 3$, which is the vacuum solution of the 4-dimensional $N=2$ massive supergravity theory.

The analysis of domain-wall solutions of other massive supergravities in $D=4$, coming from the dimensional reductions with the 2 -form or the 3 -form potential taking the extra generalised ansatz, is analogous. In the case of a toroidal compactification, the extra term 
for the 2-form potential corresponds to an harmonic 3-form that may be taken to be

$$
\begin{aligned}
\Omega_{3}= & m_{1} d z_{2} \wedge d z_{3} \wedge d z_{4}-m_{2} d z_{2} \wedge d z_{6} \wedge d z_{7}+m_{3} d z_{3} \wedge d z_{5} \wedge d z_{7} \\
& -m_{4} d z_{4} \wedge d z_{5} \wedge d z_{6} .
\end{aligned}
$$

We find that there is a solution of four intersecting 5-branes in $D=10$ if the 3 -form field strength is set equal to $\Omega_{3}$ :

$$
\begin{aligned}
& d s_{10}^{2}=\left(H_{1} H_{2} H_{3} H_{4}\right)^{-\frac{1}{4}}\left(d x^{\mu} d x^{\nu} \eta_{\mu \nu}+\left(H_{1} H_{2} H_{3} H_{4}\right) d y^{2}+\left(H_{1} H_{2}\right) d z_{2}^{2}\right. \\
&+\left(H_{1} H_{3}\right) d z_{3}^{2}+\left(H_{1} H_{4}\right)^{\frac{3}{4}} d z_{4}^{2}+\left(H_{3} H_{4}\right)^{\frac{3}{4}} d z_{5}^{2} \\
&\left.+\left(H_{2} H_{4}\right) d z_{6}^{2}+\left(H_{2} H_{3}\right) d z_{7}^{2}\right) \\
& e^{\phi_{1}=}\left(H_{1} H_{2} H_{3} H_{4}\right)^{-\frac{1}{2}}
\end{aligned}
$$

Compactification of the solution on the 6-torus gives rise to a domain wall in $D=4$, which is a solution for the 4-dimensional Lagrangian (2.30) (but with $\alpha$ now running over $1 \ldots, 4$, and $\left.\vec{c}_{1}=\vec{a}_{1234}, \vec{c}_{2}=\vec{a}_{1267}, \vec{c}_{3}=\vec{a}_{1357}, \vec{c}_{4}=\vec{a}_{1456}\right)$. When the $m_{\alpha}$ are all equal, the discussion can be extended to a generalised compactification on a Calabi-Yau manifold. Again, the requirement that the isotropic form for the internal metric should arise in the equal-charge limit demands that the 3 -form $\Omega_{3}$ have the four-term structure given in (2.32). In this case, the metric (2.33) for the solution of the four intersecting 5-branes becomes

$$
d s_{10}^{2}=H^{-1} d x^{\mu} d x^{\nu} \eta_{\mu \nu}+H^{3} d y^{2}+H d s_{6}^{2},
$$

where $d s_{6}^{2}$ is the metric for the Calabi-Yau manifold. The 3 -form $\Omega_{3}$ given by (2.32) on the 6 -torus will now be replaced by $m \Lambda$, where $\Lambda$ is the real part of the complex holomorphic 3 -form

$$
\frac{1}{6} \epsilon^{a b c} d \zeta_{a} \wedge d \zeta_{b} \wedge d \zeta_{c}
$$

where the three complex coordinates $\zeta_{a}$ can be related to the six real coordinates $z_{i}$ by $\zeta_{1}=z_{2}+\mathrm{i} z_{5}, \zeta_{2}=z_{3}+\mathrm{i} z_{6}$ and $\zeta_{3}=z_{4}+\mathrm{i} z_{7}$. Thus the structure of this holomorphic 3 -form on the Calabi-Yau space coincides with the structure that we had to choose for the harmonic 3-form 2.32) on the 6-torus in order to be able to achieve an isotropic limit for the internal metric on the torus. This is a rather striking indication that the type IIA string exhibits special features that conspire with the properties of Calabi-Yau spaces to make the construction of the associated massive supergravity possible. Similar remarks apply to the other Calabi-Yau and K3 compactifications that we discussed previously. The tendimensional solution (2.34) will reduce to a single-scalar domain-wall solution with $\Delta=1$ under the compactification that we are discussing here. 
The generalised ansatz for the 3 -form potential in a toroidal compactification corresponds to adding an harmonic 4-form to $F_{4}$ that can be taken to have the form

$$
\Omega_{4}=m_{1} d z_{2} \wedge d z_{3} \wedge d z_{4} \wedge d z_{5}+m_{2} d z_{2} \wedge d z_{3} \wedge d z_{6} \wedge d z_{7}+m_{3} d z_{4} \wedge d z_{5} \wedge d z_{6} \wedge d z_{7}
$$

The solution that survives the compactification has the 4 -form field strength equal to $\Omega_{4}$. In this case, it describes three intersecting 4-branes:

$$
\begin{aligned}
d s_{10}^{2}= & \left(H_{1} H_{2} H_{3}\right)^{-\frac{3}{8}} d x^{\mu} d x^{\nu} \eta_{\mu \nu}+\left(H_{1} H_{2} H_{3}\right)^{\frac{5}{8}} d y^{2}+\left(H_{1} H_{2}\right)^{\frac{5}{8}} H_{3}^{-\frac{3}{8}}\left(d z_{2}^{2}+d z_{3}^{2}\right) \\
& +\left(H_{1} H_{3}\right)^{\frac{5}{8}} H_{2}^{-\frac{3}{8}}\left(d z_{4}^{2}+d z_{5}^{2}\right)++\left(H_{2} H_{3}\right)^{\frac{5}{8}} H_{1}^{-\frac{3}{8}}\left(d z_{6}^{2}+d z_{7}^{2}\right) \\
e^{\phi_{1}}= & \left(H_{1} H_{2} H_{3}\right)^{\frac{1}{4}}
\end{aligned}
$$

When the $m_{\alpha}$ 's are all equal, we can extend the result to a Calabi-Yau compactification. The 10-dimensional solution (2.37), whose metric now becomes

$$
d s_{10}^{2}=H^{-9 / 8} d x^{\mu} d x^{\nu} \eta_{\mu \nu}+H^{15 / 8} d y^{2}+H^{7 / 8} d s_{6}^{2},
$$

which can be compactified on a Calabi-Yau manifold with the Ricci-flat metric $d s_{6}^{2}$, giving rise to a single-scalar domain-wall solution with $\Delta=4 / 3$ in the 4-dimensional $N=1$ massive supergravity. The 4 -form $\Omega_{4}$, which is given by (2.36) on the 6 -torus, will be replaced by $m J \wedge J$, where $J$ is the Kähler form on the Calabi-Yau space. Again, the structure of the 4-form (2.36) on the 6-torus, required in order that there exist an equal-charge limit with an isotropic metric on the 6-torus, is precisely what is needed in order to allow a generalisation to a Calabi-Yau compactification.

\section{Generalised reduction of M-theory and the type IIB string}

M-theory has only a 4-form field strength, and so a generalised dimensional reduction requires a compactification on a manifold with a non-vanishing 4'th cohomology class. The simplest generalised reduction is obtained by compactifying M-theory on a 4-manifold $M_{4}$, with the 3-form potential having an additional term whose exterior derivative is proportional to the 4 -volume form on $M_{4}$. The case of a $T^{4}$ compactification was discussed in [5], and gives rise to a $D=7, N=2$ massive supergravity, whose vacuum solution, the domain wall, is the vertical dimensional reduction of the 5 -brane in $D=11$. In [9], the discussion was extended to a generalised $\mathrm{K} 3$ compactification, giving rise to $N=1$ massive supergravity in $D=7$ with a single topological mass term for the 4-form field strength in the supergravity multiplet. This $N=1$ massive supergravity in $D=7$ was constructed 
earlier in [23], with an additional gauge parameter. The supersymmetric vacuum solution with both the gauge and mass parameters was constructed in [24].

For compactifications to $D=4$, the possibilities for the internal 7-manifold include $T^{7}$, $\mathrm{Y} \times S^{1}, \mathrm{~K} 3 \times T^{3}$, and Joyce manifolds $\mathrm{J}_{7}$, where $\mathrm{Y}$ denotes a six-dimensional Calabi-Yau manifold. Note that the generalised dimensional reductions on these manifolds have properties essentially similar to those demonstrated in the previous section. We shall therefore not discuss all the manifolds in detail, but instead we shall focus on the simplest and most illuminating example, namely the case where M-theory is compactified on a Joyce manifold. Sevendimensional Joyce manifolds are simply-connected compact manifolds with $G_{2}$ holonomy that admit Ricci-flat metrics [25]. It follows from the decomposition of the tangent-space group $S O(7)$ under the $G_{2}$ subgroup that the 8-dimensional spinor representation decomposes into $7+1$ under the holonomy group, and therefore there is one covariantly-constant Majorana spinor, $\eta$. This implies that the compactified four-dimensional supergravity theory will have $N=1$ supersymmetry. The cohomology structure of the manifold is specified by the 2'nd and 3'rd Betti numbers, with $b_{0}=1, b_{1}=0, b_{4}=b_{3}, b_{5}=b_{2}, b_{6}=0, b_{7}=1$. Included in the 3'rd cohomology is a covariantly-constant 3-form which can be constructed using $\eta$, as $\Omega_{i j k}=\bar{\eta} \Gamma_{i j k} \eta$. Its dual, $\Omega_{4}=* \Omega_{3}$, is also covariantly constant, and this is the form that we shall use for the generalised reduction of M-theory.

The bosonic Lagrangian for the low-energy limit of M-theory is

$$
\mathcal{L}=\hat{e} \hat{R}-\frac{1}{48} \hat{e} \hat{F}_{4}^{2}+\frac{1}{6} \hat{F}_{4} \wedge \hat{F}_{4} \wedge \hat{A}_{3}
$$

where $F_{4}=d A_{3}$. The generalised ansatz for the dimensional reduction of $\hat{A}_{3}$ is

$$
\hat{A}_{3}(x, z)=m \omega+A_{3}(x)+\sum_{\alpha=1}^{b_{2}} A_{1}^{\alpha}(x) \wedge \Omega_{2}^{\alpha}+\sum_{i=1}^{b_{3}} A_{0}^{(i)}(x) \Omega_{3}^{i},
$$

where $d \omega=\Omega_{4}$. Note that the dual of $\Omega_{4}$ is one of the 3 -forms $\Omega_{3}^{i}$ included in the expansion. For convenience, we may assume that it is $\Omega_{3}^{1}$. We shall choose a basis for the $\Omega_{3}^{i}$ such that $\int \Omega_{3}^{i} \wedge * \Omega_{3}^{j}=\delta^{i j}$. In addition to the 3 -form $A_{3}$, the $b_{2} 1$-form potentials $A_{1}^{\alpha}$ and $b_{3}$ axions $A_{0}^{i}$ in $D=4$, there will be $b_{L}+1$ scalars, coming from the $b_{L}$ transverse traceless zero modes of the Lichnerowicz operator on the Joyce manifold $\mathrm{J}_{7}$ together with the conformal scaling mode. These $b_{L}+1$ zero modes correspond to the independent Ricci-flat deformations of $J$. It was shown in [26] that $b_{L}$ is equal to $b_{3}-1$. Since the 4 -form field strength $\hat{F}_{4}$ in $D=11$ has no Chern-Simons corrections, the only source of mass terms in the $D=4$ theory obtained by this generalised dimensional reduction is the $\hat{F}_{4} \wedge \hat{F}_{4} \wedge \hat{A}_{3}$ term in $D=11$. In view of the fact that $\int \Omega_{4} \wedge \Omega_{3}^{i}=\delta^{1 i}$, we find that that it gives a contribution 
$\mathcal{L}_{\text {mass }}=\frac{1}{3856} m A_{0}^{(1)} \epsilon^{\mu \nu \rho \sigma} F_{\mu \nu \rho \sigma}$ to the four-dimensional Lagrangian. This term implies that the axion $A_{0}^{(1)}$ is massive. To see this, we can look at the relevant quadratic terms in the four-dimensional Lagrangian:

$$
\mathcal{L} \sim-\frac{1}{2} \sum_{i=1}^{b_{3}}\left(\partial \phi_{i}\right)^{2}-\frac{1}{2} \sum_{i=1}^{b_{3}}\left(\partial A_{0}^{(i)}\right)^{2}-\frac{1}{48} F_{4}^{2}-\frac{1}{12} \sum_{\alpha=1}^{b_{2}}\left(F_{2}^{(\alpha)}\right)^{2}+\frac{1}{3856} m A_{0}^{(1)} \epsilon^{\mu \nu \rho \sigma} F_{\mu \nu \rho \sigma}
$$

The equation of motion for $A_{3}$ implies that $F_{\mu \nu \rho \sigma}=\frac{1}{144} m A_{0}^{(1)} \epsilon_{\mu \nu \rho \sigma}$, and hence the Lagrangian for $A_{0}^{(1)}$ has the form $-\frac{1}{2}\left(\partial A_{0}^{(1)}\right)^{2}-\frac{1}{(12)^{4}} m^{2}\left(A_{0}^{(1)}\right)^{2}$.

In order to obtain the domain-wall solution in $N=1$ massive supergravity in $D=4$ that we constructed above, and to relate this solution to a solution in $D=11$ dimensions, we first consider a generalised compactification on a 7-torus. In order to be able to generalise the compactification to a Joyce manifold, it is necessary to introduce a harmonic 4-form on the 7-torus that has seven independent terms, of the form

$$
\begin{aligned}
\Omega_{4}= & m_{1} d z_{1} \wedge d z_{2} \wedge d z_{3} \wedge d z_{7}+m_{2} d z_{1} \wedge d z_{2} \wedge d z_{4} \wedge d z_{5} \\
& +m_{3} d z_{1} \wedge d z_{3} \wedge d z_{4} \wedge d z_{6}+m_{4} d z_{1} \wedge d z_{5} \wedge d z_{6} \wedge d z_{7} \\
& +m_{5} d z_{2} \wedge d z_{3} \wedge d z_{5} \wedge d z_{6}+m_{6} d z_{2} \wedge d z_{4} \wedge d z_{6} \wedge d z_{7} \\
& +m_{7} d z_{3} \wedge d z_{4} \wedge d z_{5} \wedge d z_{7}
\end{aligned}
$$

As in the previous examples in the type IIA string, the structure (3.4) is dictated uniquely, up to coordinate redefinitions, by the requirement that the associated solution have a limit where the metric on the 7-torus have an isotropic form when the charges are set equal. The solution in $D=11$, with the 4 -form field strength $\hat{F}_{4}$ equal to $\Omega_{4}$, describes seven intersecting fivebranes:

$$
\begin{aligned}
d s_{11}^{2}= & \left(H_{1} \cdots H_{7}\right)^{-\frac{1}{3}}\left(d x^{\mu} d x^{\nu} \eta_{\mu \nu}+\left(H_{1} \cdots H_{7}\right) d y^{2}+\left(H_{1} H_{2} H_{3} H_{4}\right) d z_{1}^{2}\right. \\
& \left(H_{1} H_{2} H_{5} H_{6}\right) d z_{2}^{2}+\left(H_{1} H_{3} H_{5} H_{6}\right) d z_{3}^{2}+\left(H_{2} H_{3} H_{6} H_{7}\right) d z_{4}^{2} \\
& \left.\left(H_{2} H_{4} H_{5} H_{7}\right) d z_{5}^{2}+\left(H_{3} H_{4} H_{5} H_{6}\right) d z_{6}^{2}+\left(H_{1} H_{4} H_{6} H_{7}\right) d z_{7}^{2}\right)
\end{aligned}
$$

where $H_{\alpha}=1+m_{\alpha}|y|$. Thus compactifying this solution on the 7-torus parameterised by $z^{i}$ gives rise to a domain-wall solution in $D=4$ :

$$
\begin{aligned}
d s_{4}^{2} & =\left(H_{1} \cdots H_{7}\right)^{\frac{1}{2}} d x^{\mu} d x^{\nu} \eta_{\mu \nu}+\left(H_{1} \cdots H_{7}\right)^{\frac{3}{2}} d y^{2} \\
e^{-\frac{1}{2} \vec{c}_{\alpha} \cdot \vec{\phi}} & =H_{\alpha}\left(H_{1} \cdots H_{7}\right)^{\frac{4}{3}}
\end{aligned}
$$

where

$$
\begin{array}{lll}
\vec{c}_{1}=\vec{a}_{1237}, & \vec{c}_{2}=\vec{a}_{1245}, & \vec{c}_{3}=\vec{a}_{1346}, \quad \vec{c}_{4}=\vec{a}_{1567}, \\
\vec{c}_{5}=\vec{a}_{2356}, & \vec{c}_{6}=\vec{a}_{2467}, & \vec{c}_{7}=\vec{a}_{3457} .
\end{array}
$$


Supersymmetric $p$-brane solutions with seven charges in $D=4, N=8$ supergravity were first found in [10]. When all the charge parameters $m_{\alpha}$ are equal, the metric on the 7dimensional internal space becomes proportional to $d z_{i} d z_{i}$, which can be replaced by an arbitrary Ricci-flat metric, provided that it admits a covariantly-constant 4-form that can replace the expression (3.4) on the 7-torus. Remarkably, any Joyce manifold has precisely such a 4-form. We may first consider the 3 -form dual to this, which is given, as we remarked earlier, by $\Omega_{i j k}=\bar{\eta} \Gamma_{i j k} \eta$. As is well known [27, 28], the quantities $\Omega_{i j k}$ (in a tangent-space frame) generate the multiplication table of the imaginary octonions $o_{i}$, namely $o_{i} o_{j}=$ $-\delta_{i j}+\Omega_{i j k} o_{k}$. In a suitable basis, the non-zero components of $\Omega_{i j k}$ are specified by

$$
\Omega_{147}=\Omega_{257}=\Omega_{367}=\Omega_{126}=\Omega_{234}=\Omega_{315}=\Omega_{456}=1,
$$

which, after dualising, is easily seen to give the same structure as (3.4). The 11-dimensional metric for the seven intersecting 5-branes then becomes, after setting the $m_{\alpha}$ 's equal,

$$
d s_{10}^{2}=H^{-\frac{7}{3}} d x^{\mu} d x^{\nu} \eta_{\mu \nu}+H^{\frac{14}{3}} d y^{2}+H^{\frac{5}{3}} d s_{7}^{2}
$$

where $d s_{7}^{2}$ is the Ricci-flat metric on the Joyce manifold. This solution reduces to a singlescalar domain wall with $\Delta=4 / 7$ upon compactification to $D=4$. Thus we see also in this case that M-theory has special features that are precisely what is needed in order to allow the generalised compactification on a 7-dimensional Joyce manifold.

We shall now consider generalised compactifications of the 10-dimensional type IIB theory, which, it has been argued, should be accorded a similar fundamental status to that of M-theory [29]. The bosonic fields of type IIB supergravity consist of a self-dual 5-form, two 3-form and a 1-form field strength, together with the metric and dilaton. A generalised compactification using the 1-form field strength $F_{1}=d \chi$ on a circle, with the ansatz $\chi(x, z)=m z+\chi(x)$, was first considered in [3]. It gives rise to an $N=2$ massive supergravity in $D=9$, which is T-dual to the $S^{1}$ compactification [3] of 10-dimensional $N=2$ massive supergravity 吽. In this procedure, the axion $\chi(x)$ is eaten by the KaluzaKlein vector, which becomes massive. In a compactification on an $n$-torus, the generic ansatz $\chi(x, z)=m_{i} z_{i}+\chi(x)$, where $i$ runs over the dimension of the compactified space, again generates only one cosmological term, since in the first step of reduction where a mass parameter $m_{i}$ is non-zero, the $\chi$ field is again eaten, and thus cannot generate any further cosmological terms in the subsequent reduction steps. (Put another way, the reduction gives "cosmological terms" of the form $-\frac{1}{2} m_{r}^{2} e^{\vec{c}_{r} \cdot \vec{\phi}}-\frac{1}{2} \sum_{i>r}\left(m_{i}+\chi_{i}\right)^{2} e^{\overrightarrow{c_{i}} \cdot \vec{\phi}}$, where $m_{r}$ is the first non-vanishing mass parameter, and $\chi_{i}$ are axions coming from the dimensional reduction of 
Kaluza-Klein vectors. Constant shift transformations of these axions enable the constants $m_{i}$ for $i>r$ to be absorbed, leaving only the cosmological term for $m_{r}$.) The spectrum of massive fields in 10 $-n$ dimensions does depend, however, on the value of $r$ labelling the first non-zero component $m_{r}$ of the parameters $m_{i}$ [5]. This is because each subsequent reduction step after the appearance of the massive vector potential will generate an additional massive scalar from it, and so the number of these additional massive scalars depends on the step at which the massive vector appears. In other words, ordinary Kaluza-Klein reduction and generalised Kaluza-Klein reduction do not commute in general, which was observed in the case of $S^{1}$ compactification in [5].

Generalised dimensional reductions using either of the 3-form field strengths in the type IIB theory are analogous to those that we presented for the type IIA theory. We shall not consider such a compactification on Calabi-Yau spaces further; instead, we shall consider the example of a generalised compactification to three dimensions on a 7-dimensional Joyce manifold, using the covariantly-constant 3-form $\Omega_{3}$ that we discussed previously. The NSNS 3-form $F_{3}$ and the R-R 3-form $\widetilde{F}_{3}$ are given in terms of potentials by

$$
F_{3}=d A_{2}, \quad \widetilde{F}_{3}=d \widetilde{A}_{2}+\chi d A_{2} .
$$

The trilinear term in the type IIB theory is of the form $A_{4} \wedge d A_{2} \wedge d \widetilde{A}_{2}$. This term will not give rise to any mass contributions, regardless of whether the generalised reduction is applied to $A_{2}$ or $\widetilde{A}_{2}$, since, after substituting in the Kaluza-Klein expansions it will give bilinear terms proportional to the triple-intersection numbers $\int_{J} \Omega_{2}^{i} \wedge \Omega_{2}^{j} \wedge \Omega_{3}$. Since none of the harmonic 2-forms $\Omega_{2}^{i}$ are covariantly constant, it follows that none of the harmonic 4-forms $\Omega_{2}^{i} \wedge \Omega_{2}^{j}$ are covariantly constantant, and therefore they will have zero intersection with $\Omega_{3}$. The only source of a mass term is therefore from the Chern-Simons correction in (3.10). Thus the axion $\chi$ will acquire a mass if the generalised reduction is applied to the NS-NS potential $A_{2}$, whilst it will remain massless if instead the R-R potential $\widetilde{A}_{2}$ is used. In either case, there will be a cosmological term in the 3-dimensional $N=2$ supergravity theory. The domain wall solutions of these two theories can both be oxidised back to $D=10$, becoming seven intersecting 5-branes in the type IIB theory. These carry NS-NS charges in the former case, and R-R charges in the latter.

It now remains to discuss the generalised ansatz for the self-dual 5-form, which is associated with a self-dual 3-brane in $D=10$. In fact the self-dual 3-brane can be viewed as being analogous to the M-branes of $D=11$, in that it is not coupled to the dilaton. The self-dual 3-brane solution, with its charge uniformly distributed on a 5-dimensional hyperplane in 
the transverse space, is given by

$$
\begin{aligned}
d s_{10}^{2} & =H^{-\frac{1}{2}} d x^{\mu} d x^{\nu} \eta_{\mu \nu}+H^{\frac{1}{2}}\left(d y^{2}+d s_{5}^{2}\right), \\
\hat{F}_{5} & =m\left(H^{-2} \epsilon_{4} \wedge d y+\Omega_{5}\right),
\end{aligned}
$$

where $\Omega_{5}$ is the volume form for the 5-dimensional metric $d s_{5}^{2}$, and $\epsilon_{4}$ is the volume form for the 3-brane world volume. As usual, the harmonic function $H$ takes the form $H=1+m|y|$. The flat metric $d s_{5}^{2}=d z_{i} d z_{i}$ on the hyperplane can be replaced by any Ricci-flat metric on a compactifying 5-dimensional space, giving rise to a domain wall in $D=5$. At the level of the theory itself, the compactification to a supergravity in $D=5$ that admits the domain wall solution requires a generalised ansatz of the form

$$
\hat{A}_{4}(x, z)=m \omega_{4}+\cdots
$$

where $d \omega_{4}=H^{-2} \epsilon_{4} \wedge d y+\Omega_{5}$, and the dots represent the standard harmonic expansions for the lower-dimensional massless fields. As far as we are aware, there are no particularly noteworthy 5-dimensional Ricci-flat compact spaces.

\section{Discussion and conclusions}

In this paper, we have studied the generalisations of the usual Kaluza-Klein ansatz that are necessary in order to generate lower-dimensional massive theories that admit domain wall solutions. The new characteristic of the generalised ansatz is that there are additional terms in the gauge potential, whose exterior derivatives are constant multiples of certain harmonic forms on the compactified space. As long as the gauge potential enters the higher-dimensional equations of motion everywhere via its derivative, this generalised dimensional reduction will be consistent, as in the case of standard Kaluza-Klein procedure. We focussed our attention on several such generalised reductions, for M-theory and for the type IIA and type IIB strings, compactified on certain Ricci-flat manifolds. In general, this generalised dimensional reduction procedure will give rise to a theory in the lower dimensions that contain no $p$-brane solutions with $p \leq(D-3)$. In the cases we considered, the lower-dimensional theories admit supersymmetric domain-wall solutions. Owing to the consistency of the dimensional reduction procedure, these domain-wall solutions can be oxidised back to $D=10$ or $D=11$, where they become higher-dimensional $p$-branes, or intersecting $p$-branes. A particular example worth mentioning is the generalised dimensional reduction of M-theory on a 7 -dimensional Joyce manifold $\mathrm{J}_{7}$, where the 4 -form field 
strength has an additional term that is proportional a covariantly-constant harmonic form on $\mathrm{J}$. The associated domain-wall solution in $D=4$ describes seven intersecting 5-branes upon oxidation to $D=11$, with all seven charges equal. More general solutions exist in $D=11$, where the seven charges are independent parameters. These solutions can be reduced to $D=4$ on a 7 -torus, but, as can be seen from (3.5), with a metric on the 7 -torus in which the seven coordinates enter asymmetrically. They only enter in a symmetrical way, allowing the possibility of replacing the 7 -torus metric $d z_{i} d z_{i}$ by the Ricci-flat metric on the Joyce manifold, when the charges are equal. In fact the only way of achieving a solution for intersecting 5-branes in which the seven coordinates enter symmetrically is by using exactly seven terms in the 4-form field strength, as in (3.4), with the seven charges set equal. Thus we see that special features of $D=11$ supergravity are ideally adapted for exploiting the exceptional properties of seven-dimensional Joyce manifolds.

A further issue that arises in connection with the generalised dimensional reduction discussed in this paper concerns duality. In general, the dualities that have been established in the standard Kaluza-Klein reduction cease to hold in the generalised reduction schemes. For example, the standard K3 compactification of M-theory is conjectured [30] to be related by $\mathrm{S}$ duality to the $T^{3}$ compactification of the heterotic string. This can easily be seen to be consistent at the supergravity level with the counting of the massless fields in $D=7$ corresponding to the two compactifications. In fact the two $D=7$ supergravities are related to each other by local field redefinitions, involving in particular a sign reversal of the dilaton. However, this duality seems break down if we introduce the generalised compactification procedure. To see this, note that if the 4-form field strength of $D=11$ supergravity acquires an additional term that is a constant multiple of the volume form on K3, it gives rise to a 7-dimensional supergravity with a topological mass term [9]. On the other hand, such a topological mass term cannot arise from the $T^{3}$ compactification of the heterotic theory, whether with the standard or the generalised reduction. Instead, the generalised ansatz for toroidal compactification of the heterotic supergravity will give masses to KaluzaKlein vectors, which does not happen in the generalised K3 compactification of M-theory. Similarly we find that in most other cases too, the dualities that are manifest in standard Kaluza-Klein reductions break down. One outstanding problem is that the massive type IIA supergravity in $D=10$ does not seem to be related directly to M-theory compactified in any lower dimension [5], although it was shown that its ordinary reduction on $S^{1}$ is $\mathrm{T}$ dual to the generalised reduction of massless type IIB supergravity on $S^{1}$ [3]. This leads to the conjecture in [5] that there may exist a 13-dimensional hypothetical H-theory. 
However, by contrast there are some examples where duality does seem to survive the passage to a generalised dimensional compactification. It is conjectured that the type IIA string compactified on $\mathrm{K} 3$ is dual to M-theory compactified on $\mathrm{K} 3 \times S^{1}$, using standard Kaluza-Klein reduction. At the level of supergravity, this is rather trivial, since it merely states that the $\mathrm{K} 3$ and the $S^{1}$ compactifications of 11-dimensional supergravity commute. It was shown in [9] that this commutative property is preserved even in the case of generalised dimensional reduction, where the 4 -form has an additional term proportional to the K3 volume form. This commutativity is rather non-trivial for the generalised dimensional reduction procedure. For example, it would no longer hold if we replaced the K3 compactifying manifold by $T^{4}$ [9]. In other words, M-theory compactified on $T^{4} \times S^{1}$ seems to be inequivalent to the type IIA string compactified on $T^{4}$, in the case where a generalised Kaluza-Klein ansatz is used.

Another example is provided by the generalised reduction of M-theory compactified on $\mathrm{J}_{7} \times S^{1}$. As we saw in section 3, the generalised compactification of M-theory on a 7dimensional Joyce manifold $J_{7}$ gives one massive scalar field. It is easy to show that the generalised compactification of M-theory on $\mathrm{J}_{7}$ commutes with the standard Kaluza-Klein compactification on $S^{1}$. In other words, as in the K3 case, the generalised compactication of M-theory on $\mathrm{J}^{7} \times S^{1}$ is dual to that of type IIA compactified on $\mathrm{J}_{7}$, where it is the 4 -form field strength ansätze that are generalised. It was observed that the field content of the standard Kaluza-Klein compactification of the type IIA and type IIB theories compactified on $\mathrm{J}_{7}$ are the same, which leads to the conjecture that the $\mathrm{J}_{7}$ compactification of the two theories are equivalent [31. We note that since the 3 -form and 4-form cohomology classes are Hodge dual on $\mathrm{J}_{7}$, the duality conjectured in [31] may be extended to the generalised compactification. In the type IIB theory there are two 3-form field strengths, one of which is NS-NS and the other R-R. When the NS-NS 3-form acquires an additional term that is proportional to the covariantly constant 3 -form of the $\mathrm{J}_{7}$ manifold, the resulting 3 -dimensional $N=2$ massive supergravity contains a massive scalar, which was the axionic scalar in type IIB in $D=10.2$ The field content of this $N=2$ massive supergravity in $D=3$ is identical to that from the generalised compactification of type IIA on $\mathrm{J}_{7}$, or of M-theory on $\mathrm{J}_{7} \times S^{1}$. Thus we expect that the duality of type IIA and type IIB conjectured in [31] in the context of a standard $\mathrm{J}_{7}$ compactifcation will hold even in this generalised $\mathrm{J}_{7}$ compactification. Unlike the T-duality of the type IIA and type IIB theories compactified on a circle, which is perturbative in

\footnotetext{
${ }^{2}$ Note that if we choose instead the R-R 3-form field strength to carry the extra term, the 3-dimensional theory will be $N=2$ massless supergravity, but with a cosmological term, as discussed in section 3 .
} 
all string orders, the duality of the two theories compactified on $\mathrm{J}_{7}$ is non-perturbative. This can be seen easily in the context of the generalised compactification. The domain-wall solution of the type IIA theory compactified on $\mathrm{J}_{7}$ comes from the dimensional reduction of seven intersecting $\mathrm{R}-\mathrm{R} 4$-branes in $D=10$, whilst the domain-wall solution of the type IIB theory compactified on $\mathrm{J}_{7}$ comes from the dimensional reduction of seven NS-NS 5-branes in $D=10$. The duality of the theories implies that the seven intersecting R-R type IIA 4-branes are dual to the seven intersecting NS-NS type IIB 5-branes. Dualities between NS-NS and R-R p-branes are characteristically non-perturbative.

Finally, we turn to a discussion of the supersymmetry of the domain-wall solutions in the massive supergravities that arise from the generalised dimensional reduction of M-theory or the type II theories. As we mentioned earlier, these domain-wall solutions can be oxidised back to higher dimensions, where they become intersecting $p$-branes. The fraction of supersymmetry that is preserved can then be directly calculated from the higher-dimensional supersymmetry transformation rules. This fraction of preserved supersymmetry will remain unchanged under toroidal dimensional reduction, even in the case of the generalised Kaluza-Klein ansatz that we are considering here. In fact the theory itself retains maximal supersymmetry under this reduction. On the other hand, for compactifications involving

K3, Calabi-Yau, or Joyce manifolds, the lower-dimensional theories will have $\frac{1}{2}$, $\frac{1}{4}$ or $\frac{1}{8}$ of the maximal supersymmetry respectively. The fraction of the lower-dimensional supersymmetry that the domain-wall solution preserves depends on the number of cosmological terms used in its construction. Although the domain-wall solution may become a multi-charge intersecting $p$-brane solution in the higher dimension, it is nevertheless a single-charge solution in the lower dimensional theory obtained by K3, Calabi-Yau or Joyce manifold compactification, and hence it preserves half of the lower-dimensional supersymmetry.

\section{References}

[1] C.N. Pope and K.S. Stelle, Zilch currents, supersymmetry and Kaluza-Klein consistency, Phys. Lett. B198 (1987) 151.

[2] M.J. Duff, S. Ferrara, C.N. Pope and K.S. Stelle, Massive Kaluza-Klein modes with effective theories of superstring moduli, Nucl. Phys. B333 (1990) 783.

[3] E. Bergshoeff, M. de Roo, M.B. Green, G. Papadopoulos and P.K. Townsend, Duality of type II 7-branes and 8-branes, Nucl. Phys. B470 (1996) 113. 
[4] L.J. Romans, Massive $N=2 a$ supergravities in ten dimensions, Phys. Lett. B169 (1986) 374 .

[5] P.M. Cowdall, H. Lü, C.N. Pope, K.S. Stelle and P.K. Townsend, Domain walls in massive supergravities, hep-th/9608173, to appear in Nucl. Phys. B.

[6] J. Scherk and J.H. Schwarz, How to get masses from extra dimensions, Nucl. Phys. B153 (1979) 61.

[7] M.J. Duff and R.R. Khuri, Four-dimensional string/string duality, Nucl. Phys. B411 (1994) 473.

[8] M.J. Duff, R.R. Khuri, R. Minasian and J. Rahmfeld, New black hole, string and membrane solutions of the four-dimensional heterotic string, Nucl. Phys. B418 (1994) 195.

[9] H. Lü and C.N. Pope, Domain walls from M-branes, hep-th/9611079.

[10] H. Lü and C.N. Pope, p-brane solitons in maximal supergravities, Nucl. Phys. B465 (1996) 127.

[11] G. Papadopoulos and P.K. Townsend, Intersecting M-branes, Phys. Lett. B380 (1996) 273.

[12] A.A. Tseytlin, Harmonic superpositions of M-branes, Nucl. Phys. B475 (1996) 149.

[13] I.R. Klebanov and A.A. Tseytlin, Intersecting M-branes as four-dimensional black holes, Nucl. Phys. B475 (1996) 179.

[14] J.P. Gauntlett, D.A. Kastor and J. Traschen, Overlapping branes in M-theory, hepth/9604179.

[15] N. Khviengia, Z. Khviengia, H. Lü and C.N. Pope, Intersecting M-branes and bound states, Phys. Lett. B388 (1996) 21.

[16] H. Lü, C.N. Pope and K.S. Stelle, Vertical versus diagonal dimensional reduction for p-branes, hep-th/9605082.

[17] H. Lü, C.N. Pope, E. Sezgin and K.S. Stelle, Stainless super p-branes, Nucl. Phys. B456 (1995) 669. 
[18] M. Cvetic, S. Griffies and S-J. Rey, Static domain walls in supergravities, Nucl. Phys. B381 (1992) 301.

[19] G.W. Gibbons, Global structure of supergravity domain wall spacetimes, Nucl. Phys. B394 (1993) 3.

[20] M. Cvetic, Extreme domain wall — black hole complementary in $N=1$ supergravity with a general dilaton coupling, Phys. Lett. B341 (1994) 160.

[21] J. Polchinski and E. Witten, Evidence for heterotic type I string duality, Nucl. Phys. B460 (1996) 525.

[22] H, Lü, C.N. Pope and P.K. Townsend, Domain walls from anti-de Sitter spacetime, hep-th/9607164, to appear in Phys. Lett. B.

[23] L. Mezincescu, P.K. Townsend and P. van Nieuwenhuizen, Stability of gauged $D=7$ supergravity and the definition of masslessness in AdS in seven-dimensions, Phys. Lett. B143 (1984) 384.

[24] H. Lü, C.N. Pope, E. Sezgin and K.S. Stelle, Dilatonic p-brane solitons, Phys. Lett. B371 (1996) 46.

[25] D.D. Joyce, Compact Riemannian 7-manifolds with $G_{2}$ holonomy:1;2, J. Diff. Geom. 43 (1996) 291; 329.

[26] G.W. Gibbons, D.N. Page and C.N. Pope, Einstein metrics on $S^{3}, R^{3}$ and $R^{4}$ bundles, Comm. Math. Phys. 127 (1990) 529.

[27] F. Gürsey and C-H. Tze, Octonionic torsion on $S^{7}$ and Englert's compactification of $d=11$ supergravity, Phys. Lett. B127 (1983) 191.

[28] F. Englert, M. Rooman and P. Spindel, Symmetries in eleven-dimensional supergravity compactified on a parallelized seven-sphere, Phys. Lett. B130 (1983) 50.

[29] C. Vafa, Evidence for F theory, Nucl. Phys. B469 (1996) 403.

[30] E. Witten, String theory dynamics om various dimensions, Nucl. Phys. B443 (1995) 85.

[31] G. Papadopoulos and P.K. Townsend, Compactification of $D=11$ supergravity on space of exceptional holonomy, Phys. Lett. B357 (1995) 300. 